\title{
Proposta de uma Função de Janela com Parâmetros Ajustáveis Baseada nos Polinômios de Jacobi com Aplicação no Sistema f-OFDM
}

\author{
Maykon Renan Pereira da Silva, Flávio Geraldo Coelho Rocha e Hudson Henrique de Souza Lopes
}

\begin{abstract}
Resumo-Durante muito tempo, a técnica de janelamento tem sido utilizada em aplicações reais envolvendo o sistema de Multiplexação por Divisão de Frequência Ortogonal (OFDM), como solução para resolver um dos grandes problemas desta tecnologia, a Emissão Fora de Banda (OOBE). Por este motivo, propõese uma nova função de janela com parâmetros ajustáveis para aplicação no sistema f-OFDM (OFDM filtrado), com objetivo de reduzir a OOBE desta tecnologia. A função de janela proposta é baseada nos polinômios de Jacobi utilizando o método da série de Fourier para a estimar os seus coeficientes. A janela Jacobi tem como vantagem em relação às janelas Hanning $e$ Nakagami possuir uma menor largura de lóbulo principal para um mesmo valor de amplitude máxima do lóbulo lateral, o que garante uma menor distorção do formato do pulso. Já em relação às janelas Kaiser $e$ Dolph-Chebyshev a janela proposta tem a vantagem de ter uma maior taxa de atenuação dos lóbulos laterais, consequentemente, o sinal f-OFDM utilizando a função de janela Jacobi concentra mais potência no lóbulo principal. Os resultados obtidos por meio de simulações mostram que a janela proposta devido à flexibilidade dos seus parâmetros, quando aplicada no sistema f-OFDM, reduz a OOBE e consequentemente aumenta eficiência espectral.
\end{abstract}

Palavras-Chave-Eficiência Espectral, f-OFDM, Funções de Janela, OOBE, Polinômios de Jacobi.

Abstract-For a long time, the windowing technique has been used in real applications involving the Orthogonal Frequency Division Multiplexing (OFDM) system, as a solution to solve one of the great problems of this technology, the Out-of-Band Emission (OOBE). For this reason, a new window function with adjustable parameters is proposed for application in the $\mathrm{f}-\mathrm{OFDM}$ (filtered-OFDM) system, to reduce the OOBE of this technology. The proposed window function is based on Jacobi's polynomials using the Fourier series method to estimate its coefficients. The Jacobi window has the advantage over the Hanning and Nakagami windows in that it has a smaller main lobe width for the same maximum side lobe level, which guarantees less distortion of the pulse shape. About the Kaiser and DolphChebyshev windows, the proposed window has the advantage of having a side lobe fall-off rate, consequently, the f-OFDM signal using the Jacobi window function concentrates more power in the main lobe. The results obtained through simulations show that the proposed window due to the flexibility of its parameters, when applied to the f-OFDM system, reduces OOBE and consequently increases spectral efficiency.

Keywords-Spectral Efficiency, f-OFDM, Window Functions,

Maykon R.P. da Silva, Flávio G.C. Rocha e Hudson H.S. Lopes fazem parte do Grupo INCOMM (Information and Communication Engineering Group) da Escola de Engenharia Elétrica, Mecânica e de Computação (EMC), Universidade Federal de Goiás (UFG), Goiânia, Goiás, Brasil, e-mail: maykonrenan@discente.ufg.br, flaviogcr@ufg.br, hudson.hs1@gmail.com.

$\mathrm{O}$ presente trabalho foi realizado com apoio da Coordenação de Aperfeiçoamento de Pessoal de Nível Superior -Brasil (CAPES) - Código de Financiamento 001
OOBE, Jacobi Polynomials.

\section{INTRODUÇÃO}

Funções de janela tem sido propostas com o objetivo de atender às mais diversas aplicações [1] -[3]. Nos últimos anos, as funções de janela tem sido constantemente descritas e aplicadas em trabalhos envolvendo sistemas de modulação ortogonal, como por exemplo, UFMC (Universal Filtered MultiCarrier) e OFDM (Orthogonal Frequency Division Multiplexing) [4]-[8]. De fato, estudos têm mostrado que algumas tecnologias de modulação para sistemas de comunicação sem fio de última geração podem apresentar baixa Emissão Fora de Banda (OOBE - Out-Of-Band Emission) do sinal transmitido quando comparadas à tecnologia OFDM convencional pelo fato de implantarem janelamento e/ou filtragem na forma de onda transmitida, como por exemplo, o sistema f-OFDM (filtered-OFDM) [5], [6], [9].

Este artigo propõe uma função de janela com parâmetros ajustáveis baseada nos polinômios de Jacobi, que será aplicada no sistema f-OFDM, com o objetivo de reduzir a OOBE, e consequentemente aumentar a eficiência espectral desta tecnologia. O desempenho da função de janela proposta, quando aplicada no sistema f-OFDM, será analisado no domínio da frequência: avaliando a Densidade Espectral de Potência (PSD - Power Spectral Density) onde os níveis de OOBE poderão ser observados, Eficiência Espectral (Spectral Efficiency) e Relação Sinal-Interferência (SIR - Signal-to-Interference Ratio).

O restante do artigo está organizado da seguinte forma: A Seção II apresenta o funcionamento e as características dos sistemas de modulação OFDM convencional e f-OFDM; A Seção III apresenta as características das funções de janela e seus parâmetros espectrais; A Seção IV apresenta as funções de janela clássicas da literatura e suas aplicações em redes de comunicações móveis; A Seção V]apresenta a função de janela proposta baseada nos polinômios de Jacobi e sua respectiva demonstração; A Seção VI apresenta o desempenho da função de janela proposta em termos da análise espectral e através da aplicação no sistema f-OFDM; Na Seção VII são apresentadas as conclusões obtidas.

\section{Sistemas de Modulação OFDM E F-OFDM}

Embora o sistema OFDM tenha sido adotado como forma de onda de transmissão em sistemas com e sem fio há anos, essa tecnologia possui algumas limitações que podem tornála um recurso legado para uso em determinadas aplicações 
envolvendo as redes móveis de quinta geração (5G). A principal candidata do 3GPP (3rd Generation Partnership Project) é uma nova versão do sistema OFDM, chamada f-OFDM, que é semelhante ao OFDM, mas com filtragem adicional (utiliza o filtro sinc-janelado) para reduzir as emissões fora de banda e obter uma melhor localização espectral [10]-[12].

\section{A. $O F D M$}

A ideia principal do sistema OFDM utilizado em comunicações sem fio é dividir a largura de banda de transmissão em várias subportadoras ortogonais [9]. Um sinal OFDM geral pode ser representado como:

$$
s(t)=\sum_{n=-\infty}^{\infty} s_{n}\left(t-n T_{f}\right),
$$

onde $T_{f}$ é a duração do símbolo OFDM e $s_{n}(t)$ é o $n$-ésimo símbolo OFDM do slot downlink, dado pela seguinte equação:

$$
\begin{aligned}
s_{n}(t) & =\sum_{k=0}^{K-1} a_{n, k} \delta(t) \otimes v(t) e^{j 2 \pi k \Delta f t} \\
& =\sum_{k=0}^{K-1} a_{n, k} v(t) e^{j 2 \pi k \Delta f t},
\end{aligned}
$$

onde $\otimes$ representa a operação de convolução, $\delta(t)$ representa a função impulso, $k$ é o índice da subportadora, $K$ é o número de subportadoras, $v(t)$ denota o formato do pulso (pulse shape), $a_{n, k}$ é o conjunto de dados complexos da $k$-ésima subportadora e do $n$-ésimo símbolo OFDM e $\Delta f$ denota o intervalo entre subportadoras. No sistema OFDM convencional, $v(t)$ é um pulso retangular com largura $T_{f}$. Portanto, com base nas equações (1) e (2), o sinal OFDM é dado por:

$$
s(t)=\sum_{k=0}^{K-1} a_{n, k} v\left(t-n T_{f}\right) e^{j 2 \pi k \Delta f\left(t-n T_{f}\right)} .
$$

No sistema OFDM, após um processo de modulação (que também é chamado de mapeamento de símbolos), os símbolos de dados modulados serão convertidos de um fluxo serial para um fluxo paralelo. Posteriormente, aplica-se a IFFT (Inverse Fast Fourier Transform), onde os dados modulados serão transportados por subportadoras OFDM. Posteriormente, o fluxo paralelo será transformado em um fluxo serial e convertido para a frequência de transmissão. Existem diferentes variações do OFDM convencional. A fim de evitar Interferência Intersimbólica (ISI - Intersymbol Interference), um intervalo de guarda, como Prefixo Cíclico (CP - Cyclic Prefix) pode ser inserido entre símbolos OFDM [9].

Para o sistema OFDM convencional, $v(t)$, tal que $\mathscr{F}\{v(t)\}=V(f)$ pode ser expresso como:

$$
V(f)=T_{f} \operatorname{sinc}\left(T_{f} f\right)=\frac{T_{f} \operatorname{sen}\left(\pi T_{f} f\right)}{\pi T_{f} f} .
$$

A PSD para o sinal OFDM, tal que $\mathscr{F}\{s(t)\}=S(f)$ é descrita como:

$$
S(f)=\sigma_{a}^{2} T_{f} \sum_{k=0}^{K-1}\left|\operatorname{sinc}\left[T_{f}(f-k \Delta f)\right]\right|^{2},
$$

onde $\sigma_{a}^{2}$ é o desvio padrão de $a_{n, k}$.

\section{B. $f$-OFDM}

A transmissão do sinal em um sistema f-OFDM é muito semelhante ao OFDM, diferenciando-se apenas pela introdução de um filtro passa baixa. Uma opção de filtro é aquele com resposta ao impulso dada por uma função do tipo sinc [5], [6]. Esta função no domínio do tempo produz no domínio da frequência a resposta de um filtro passa baixa ideal. Todas as frequências abaixo da frequência de corte passam a ter amplitude unitária, enquanto as outras frequências são simplesmente bloqueadas. Porém, em termos computacionais, é impossível filtrar um sinal por um tempo infinito. Truncar a função sinc, limitando-a no domínio do tempo, não é uma solução interessante, pois a resposta em frequência dessa função apresentará bastante ripple (ondulação) devido a descontinuidade abrupta da função sinc promovida pelo processo de truncagem (efeito Gibbs) [13]. Uma forma de limitar e ao mesmo tempo suavizar a transição da função sinc para zero é multiplicá-la por uma função de janela. Logo, define-se o filtro sinc-janelado para o sistema f-OFDM:

$$
h(l)=\frac{p(l) \cdot w(l)}{\sum_{i} p(i) \cdot w(i)}
$$

e,

$$
p(l)=\operatorname{sinc}\left(\Delta f(K+2 R) \frac{l}{N_{i f f t}}\right),
$$

para $-L / 2 \leq l \leq L / 2$, onde $L$ é a ordem do filtro, $R$ denota o excesso de largura de banda e $N_{i f f t}$ é a ordem da IFFT [14].

Logo, o sinal f-OFDM $(\tilde{s}(t))$ é obtido aplicando o filtro normalizado $h(l)$ no sinal OFDM (descrito por (3) ) em cada sub-banda, da seguinte maneira:

$$
\begin{aligned}
\tilde{s}(t) & =\sum_{n=-\infty}^{\infty} \tilde{s}_{n}\left(t-n T_{f}\right) \\
& =\left[\sum_{k=0}^{K-1} a_{n, k} v\left(t-n T_{f}\right) e^{j 2 \pi k \Delta f\left(t-n T_{f}\right)}\right] \otimes h\left(t-n T_{f}\right) .
\end{aligned}
$$

A PSD do sinal f-OFDM, tal que $\mathscr{F}\{\tilde{s}(t)\}=\tilde{S}(f)$ é dada por:

$$
\tilde{S}(f)=|H(f)|^{2} \sigma_{a}^{2} T_{f} \sum_{k=0}^{K-1}\left|\operatorname{sinc}\left[T_{f}(f-k \Delta f)\right]\right|^{2},
$$

onde $H(f)$ representa o filtro sinc-janelado no domínio da frequência, tal que:

$$
H(f)=\left|\operatorname{rect}\left(\frac{f}{2 B}\right) \otimes W(f)\right|^{2},
$$

onde $B$ representa a largura de banda e $W(f)$ é a função de janela no domínio da frequência, logo:

$$
\tilde{S}(f)=\left|\operatorname{rect}\left(\frac{f}{2 B}\right) \otimes W(f)\right|^{2} \sigma_{a}^{2} T_{f} \sum_{k=0}^{K-1}\left|\operatorname{sinc}\left[T_{f}(f-k \Delta f)\right]\right|^{2} \text {. }
$$

No esquema f-OFDM, a PSD de toda a sub-banda torna-se bem localizada, pois o sinal do sistema OFDM passou através de um filtro bem localizado em frequência, cuja largura de banda está próxima da largura de banda da sub-banda. Como resultado, apenas as subportadoras próximas às bordas são 
afetadas pelo filtro, uma vez que o filtro reduz a potência dos seus lóbulos laterais. Além disso, o sistema f-OFDM também fornece reduções significativas no uso da Banda de Guarda (GB - Guard Band), levando a uma utilização mais eficiente do espectro [5], [9], [15].

\section{TÉCNICA DE JANElAMENTo E SEUS PARÂMETROS ESPECTRAIS}

Uma função de janela tem como objetivo minimizar os efeitos do Vazamento Espectral (Spectral Leakage), com o objetivo de obter uma melhor representação do espectro de frequência do sinal. A resposta em frequência de uma função de janela é composta pelo lóbulo principal e pelos lóbulos laterais [13], [16].

A Fig. 1 apresenta o gráfico da PSD da função de janela Retangular. Além disso, são apresentados alguns parâmetros espectrais que são amplamente utilizados como métrica de desempenho na análise espectral, além de auxiliar na seleção da janela apropriada para uma aplicação específica. Os parâmetros das funções da janela que são geralmente usados para a avaliação são os seguintes [16]: HMLW (Half Main Lobe Width) é a frequência em que a amplitude do lóbulo principal, ao decair, se torna igual a maior amplitude dos lóbulos laterais; MSLL (Maximum Side Lobe Level) é a amplitude máxima do lóbulo lateral; SLFOR (Side Lobe Fall-Off Rate) é a taxa de atenuação dos lóbulos laterais.

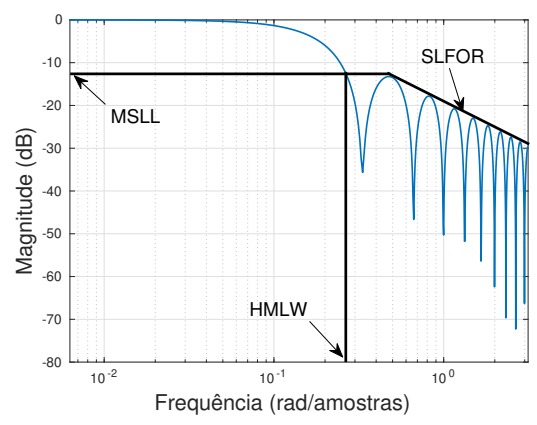

Fig. 1

DENSIDADE ESPECTRAL DE POTÊNCIA DA JANELA RETANGULAR E SEUS PARÂMETROS ESPECTRAIS.

\section{FunÇÕes de Janela e ApliCaÇões em Esquemas DE MOdULAÇÃo ORTOGONAL}

A função janela Hanning é um caso especial da função cosseno levantado [13], [16]. A janela Hanning é considerada uma boa escolha para ser utilizada na análise espectral de sinais, já que, tem como principal característica, uma elevada SLFOR, o que contribui na redução do vazamento espectral. A janela Hanning é frequentemente utilizada em aplicações envolvendo o sistema f-OFDM, pois essa função de janela, quando aplicada nesta tecnologia, consegue de maneira eficiente diminuir a OOBE, o que reduz o uso da banda de guarda, aumentando a eficiência espectral [5], [6], [10], [15].

Em [17] foi proposta a função de janela Kaiser baseada nas funções de Bessel. Devido a sua flexibilidade, a janela Kaiser é utilizada na análise espectral e para projetar filtros não-recursivos. Ao ajustar seus dois parâmetros independentes, pode-se controlar alguns parâmetros espectrais, como a HMLW e MSLL para várias aplicações. Recentemente, a janela Kaiser foi utilizada em um modelo de otimização de parâmetros do filtro sinc-janelado em um sistema uplink fOFDM multiusuário, com o objetivo de aumentar a eficiência espectral desta tecnologia [7].

Em [18] foi proposta uma função de janela ajustável baseada nos polinômios de Chebyshev. A função de janela DolphChebyshev tem como principal característica, no domínio da frequência, ter todos os seus lóbulos laterais com a mesma amplitude, ou seja, SLFOR $=0 \mathrm{~dB}$. Além disso, os filtros projetados usando essa janela tem como vantagem possuir uma banda de transição estreita. Por este motivo, esta função de janela é frequentemente citada e utilizada em aplicações envolvendo o sistema de modulação ortogonal UFMC, com o objetivo de reduzir a OOBE [4], [10], [12].

Em [19] foi proposto um algoritmo para obter uma função de janela e um par de filtros "ótimos". Para tanto, os autores utilizaram o Método de Pontos Interiores (InteriorPoint Method). Posteriormente, foi proposta uma variação do sistema de modulação OFDM utilizando janelamento e filtragem conjunta, com o objetivo de atenuara OOBE e consequentemente aumentar a eficiência espectral.

Em [20] foi proposta uma função de janela ajustável baseada na distribuição de Nakagami com o intuito de aplicá-la ao filtro sinc-janelado no sistema f-OFDM. Através do ajuste do seus parâmetros, a janela de Nakagami apresentou resultados interessantes para PSD, reduzindo a OOBE e a potência nos lóbulos laterais do sinal OFDM.

\section{Proposta de FunÇão de Janela Baseada nos POLINÔMIOS DE JACOBI}

Nesta seção apresenta-se a Proposição 1, e sua respectiva Demonstração, que fundamentam a abordagem proposta. A função de janela proposta baseia-se nos polinômios ortogonais de Jacobi $P_{m}^{\alpha, \beta}(\theta)$. Utilizando os polinômios de Jacobi é possível construir uma janela capaz de controlar a SLFOR, sendo uma vantagem em relação às janelas de Dolph-Chebyshev, Kaiser e Nakagami. A partir da propriedade de ortogonalidade, pode-se determinar recursivamente os polinômios de Jacobi:

$$
\begin{aligned}
& P_{m}^{\alpha, \beta}(\theta)=\frac{1}{2 m(m+\alpha+\beta) \bar{P}_{2}} \\
\times \quad & \left\{\bar{P}_{1}\left[(2 m+\alpha+\beta) \bar{P}_{2} \theta+\alpha^{2}-\beta^{2}\right] P_{m-1}^{\alpha, \beta}(\theta)\right. \\
- & \left.2(m+\alpha-1)(m+\beta-1)(2 m+\alpha+\beta) P_{m-2}^{\alpha, \beta}(\theta)\right\},
\end{aligned}
$$

onde $m$ representa o grau do polinômio, e $\alpha$ e $\beta$ são os expoentes do polinômio de Jacobi, $\bar{P}_{1}=2 m+\alpha+\beta-1$ e $\bar{P}_{2}=2 m+\alpha+\beta-2$. Para $m=0$ e $m=1$, os polinômios de Jacobi são dados por, $P_{0}^{\alpha, \beta}(\theta)=1$ e $P_{1}^{\alpha, \beta}(\theta)=(\alpha+1)+$ $(\alpha+\beta+2) \frac{\theta-1}{2}$, respectivamente [21]. Posteriormente, utilizase o método da série de Fourier para estimar os coeficientes da função de janela proposta. Este método tem sido utilizado em trabalhos na elaboração de funções de janela baseadas em polinômios ortogonais [18], [22]. 
Proposição 1: A função de janela Jacobi é descrita em função dos coeficientes que compõem a série de Fourier. A abordagem proposta possui quatro parâmetros independentes $\left(\alpha, \beta, x_{0}\right.$ e $\left.M\right): \alpha$ e $\beta$ são os parâmetros responsáveis por controlar a amplitude dos lóbulos laterais; $x_{0}$ controla a taxa de atenuação dos lóbulos laterais; e $M$, o comprimento da função de janela, é responsável por ajustar a largura do lóbulo principal. A função de janela Jacobi é dada por:

$$
\begin{aligned}
& \hat{w}(l)=\frac{1}{2 M+1} \\
& \times\left\{1+2 \xi \sum_{x=1}^{M} P_{2 M}^{\alpha, \beta}\left(x_{0} \cos \frac{2 \pi x}{2 M+1}\right)\left(1+\cos \frac{2 \pi x l}{2 M+1}\right)\right\},
\end{aligned}
$$

onde $\xi=1 / P_{2 M}^{\alpha, \beta}\left(x_{0}\right), \alpha, \beta \geq 1, x_{0}$ está contido no intervalo $[-1,1]$ e $|l| \leq M$.

Demonstração: Aproximando-se os polinômios de Jacobi pelo método da série de Fourier, pode-se descrever os polinômios da seguinte maneira:

$$
P_{m}^{\alpha, \beta}(\theta)=\frac{w(0)}{2}+\sum_{l=1}^{T_{w}-1} w(l) \cos \frac{2 \pi x l}{T_{w}}
$$

onde $\theta=x_{0} \cos \frac{2 \pi x}{T_{w}}, T_{w}=2 M+1$ e $w(l)$ representa os coeficientes da série de Fourier, que variam de acordo com a função $P_{m}^{\alpha, \beta}(\theta)$ por um período $T_{w}$. Os coeficientes $w(l)$ podem ser calculados pela seguinte equação:

$$
\begin{aligned}
w(l) & =\frac{1}{T_{w}} \int_{0}^{T_{w}} P_{m}^{\alpha, \beta}(\theta) d x+\frac{1}{T_{w}} \int_{0}^{T_{w}} P_{m}^{\alpha, \beta}(\theta) \cos \frac{2 \pi x l}{T_{w}} d x \\
& =\frac{1}{T_{w}} \int_{0}^{T_{w}} P_{m}^{\alpha, \beta}(\theta)\left(1+\cos \frac{2 \pi x l}{T_{w}}\right) d x .
\end{aligned}
$$

Como $x$ representa valores de tempo em instantes discretos, os coeficientes $w(l)$ podem ser obtidos por meio da transformada de Fourier de tempo discreto, da seguinte maneira:

$$
\begin{aligned}
w(l) & =\frac{1}{T_{w}} \sum_{x=0}^{2 M} P_{2 M}^{\alpha, \beta}(\theta)\left(1+\cos \frac{2 \pi x l}{T_{w}}\right) \\
& =\frac{1}{T_{w}}\left\{2 \sum_{x=0}^{M-1} P_{2 M}^{\alpha, \beta}(\theta)\left(1+\cos \frac{2 \pi x l}{T_{w}}\right)\right\} .
\end{aligned}
$$

Quando $x=0$, obtém-se $\theta=x_{0}$. Logo, rearranjando-se os termos em 16, obtém-se:

$$
w(l)=\frac{1}{2 M+1}\left\{P_{2 M}^{\alpha, \beta}\left(x_{0}\right)+2 \sum_{x=1}^{M} P_{2 M}^{\alpha, \beta}(\theta)\left(1+\cos \frac{2 \pi x l}{T_{w}}\right)\right\} .
$$

Substituindo o parâmetro $\theta$ e o período $T_{w}$ e dividindo $w(l) / P_{2 M}^{\alpha, \beta}\left(x_{0}\right)$, obtém-se a função de janela Jacobi normalizada $(\hat{w}(l))$, dada por 13 , como se queria demonstrar.

A função de janela Jacobi no domínio da frequência, tal que $\mathscr{F}\{\hat{w}(l)\}=\hat{W}(f)$ (onde $\mathscr{F}\{\cdot\}$ representa a transformada de Fourier ) é dada pela seguinte equação:

$$
\hat{W}(f)=\frac{P_{2 M}^{\alpha, \beta}\left[x_{0} \cos (f / 2)\right]}{P_{2 M}^{\alpha, \beta}\left(x_{0}\right)} .
$$

A função de janela Jacobi tem como vantagem em relação às janelas Hanning e Nakagami possuir uma HMLW 10\% a 20\% menor, para um mesmo valor de MSLL, o que garante uma menor distorção do formato do pulso, reduzindo a OOBE e garantindo uma maior eficiência espectral. A SLFOR da janela Jacobi varia de $3 \mathrm{~dB} /$ oitava até $20 \mathrm{~dB} /$ oitava (dependendo do valor de $x_{0}$ ), enquanto a janela Kaiser tem uma SLFOR de $6 \mathrm{~dB} /$ oitava e a janela Dolph-Chebyshev de 0 dB/oitava. Consequentemente, o sinal f-OFDM utilizando a função de janela Jacobi concentra mais potência no lóbulo principal, já que possui uma alta atenuação dos lóbulos laterais. Logo, o sinal f-OFDM tende a ter uma maior SIR, garantindo uma maior qualidade na comunicação móvel.

\section{RESUltados}

Nesta seção, será avaliado o desempenho do sistema fOFDM utilizando a função de janela Jacobi, através da PSD onde poderá ser observado os níveis de OOBE, eficiência espectral e SIR. Além disso, o desempenho da janela proposta será comparado ao da função de janela Hanning. No cenário de simulação do sistema f-OFDM foram utilizados os seguintes parâmetros: $K=24$ subportadoras, $\Delta f=15 \mathrm{kHz}, \mathrm{CP}=72$, $L=100$ (ordem do filtro), $N_{i f f t}=1024$ (ordem da IFFT), utilizando a modulação 64-QAM e canal com desvanecimento Rayleigh. Os resultados foram obtidos com uma média de 100 simulações.

As Fig. 2a e 2p apresentam a resposta ao impulso do filtro sinc, e dele mesmo multiplicado com a janela Jacobi. Percebese que, ao multiplicar a função sinc pela janela proposta, há uma redução da amplitude lóbulos laterais da resposta ao impulso do filtro ao longo de tempo, principalmente quando aumenta-se o valor de $\beta$ e tem-se $x_{0}=1$. Este comportamento tem reflexo na resposta em frequência do filtro sinc-janelado, como mostra as Fig. 2k e 2d. Nota-se que, para $x_{0}=1$ (Fig. 2.) o filtro apresenta em sua banda de rejeição uma maior atenuação dos seus lóbulos laterais, ou seja, essa configuração de filtro quando aplicada no sinal OFDM, tenderá a concentrar mais potência do sinal no lóbulo principal, quando comparado às configurações do filtro da Fig. $2 \mathrm{~d}$, onde $x_{0}=-1$. Entretanto, para $x_{0}=-1$, a banda de transição do filtro é ligeiramente mais estreita na Fig. 2 d, neste caso, quando aplicado no sinal OFDM tem-se uma menor distorção do formato do pulso do sinal, além disso, essa distorção cresce, a medida que $\beta$ aumenta, como é possível perceber na PSD mostrada nas Fig. 2e e 2f. Nota-se nas Fig. 2e e 2f, como a aplicação do filtro no sinal OFDM reduz a OOBE em relação ao mesmo sem filtragem. Entretanto, analisando o sinal f-OFDM (janela Jacobi), quando $\beta \rightarrow \infty$ a MSLL $\rightarrow-\infty$ (mais potência no lóbulo principal), porém a HMLW $\rightarrow \infty$ (maior distorção do formato do pulso). Pode-se solucionar essa distorção do pulso do sinal f-OFDM aumentando a ordem do filtro $(L)$, entretanto, mais complexidade seria adicionada ao sistema f-OFDM. O reflexo disso pode ser observado nas Fig. $2 \mathrm{~g}$ e 2 h. Nota-se na Fig. 2g, que uma menor distorção do pulso aumenta a eficiência espectral do sistema f-OFDM, pois reduz os níveis de OOBE e introdução de intervalos de guarda. Entretanto, se a MSLL $\rightarrow-\infty$ ou a SLFOR $\rightarrow \infty$, obtém-se uma maior relação entre a potência do sinal f-OFDM e a interferência oriunda do aumento no deslocamento do tempo de símbolo 
OFDM, como mostra a Fig. 2h. Conclui-se que, a janela Jacobi devido a flexibilidade do ajuste dos seus parâmetros torna-se uma solução interessante para o sistema f-OFDM, já que, pode adaptar-se a uma determinada aplicação que necessita de uma maior eficiência espectral ou outro serviço que requisita mais potência no sinal f-OFDM a ser transmitido.

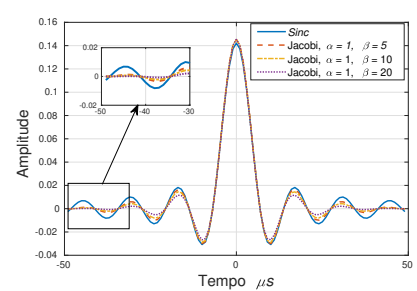

(a)

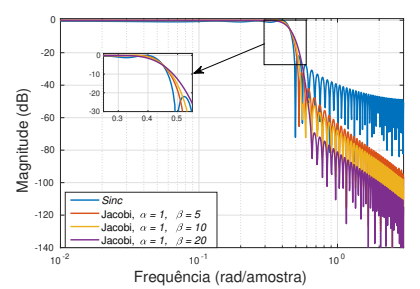

(c)

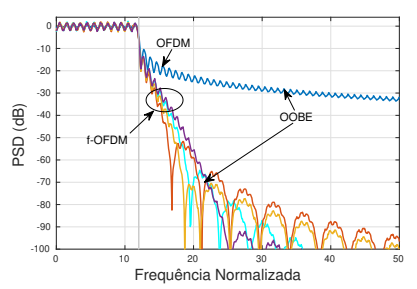

(e)

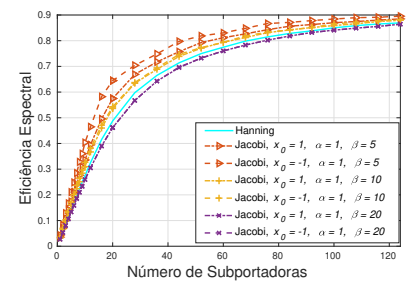

(g)

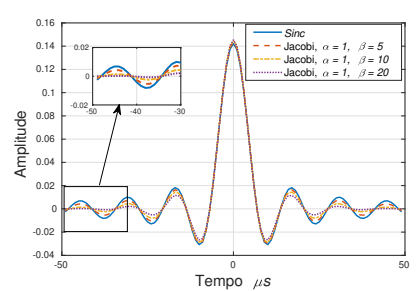

(b)

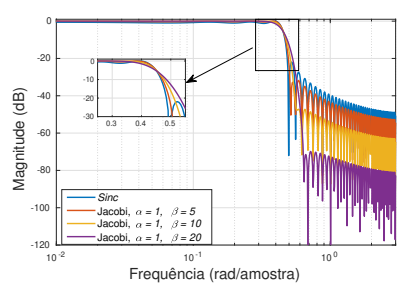

(d)

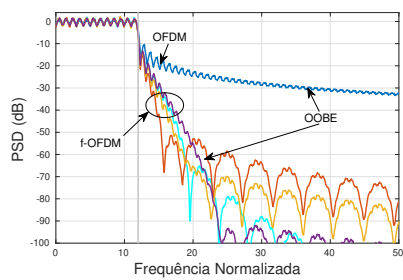

(f)

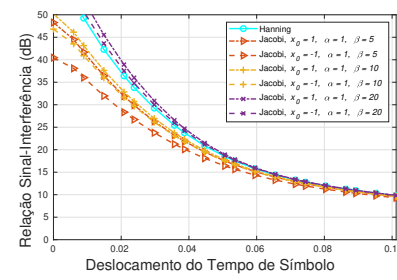

(h)
Fig. 2

A) Resposta ao impulso do filtro, $x_{0}=1$. B) Resposta Ao impulso DO FILTRO, $x_{0}=-1$. C) RESPOSTA EM FREQUÊNCIA FILTRO, $x_{0}=1$. D) RESPOSTA EM FREQUÊNCIA FILTRO, $x_{0}=-1$. E) PSD DO SINAL OFDM E

F-OFDM, $x_{0}=1$. F) PSD DO SINAL OFDM E F-OFDM, $x_{0}=-1$. G)

EFICIÊNCIA ESPECTRAL versus NÚMERO DE SUBPORTADORAS $(K)$. H) SIR versus DESLOCAMENTO DO TEMPO DE SÍMBOLO.

\section{CONCLUSÃO}

Este trabalho propôs uma nova janela ajustável baseada nos polinômios de Jacobi com o intuito de aplicá-la ao filtro sinc-janelado do sistema f-OFDM. A janela Jacobi apresentou resultados promissores para $\mathrm{PSD}$, reduzindo a OOBE e aumentando a eficiência espectral do sistema f-OFDM, além disso, mostrou-se ser uma janela com desempenho superior à janela Hanning, devido a flexibilidade do ajuste de seus parâmetros. Para trabalhos futuros, pretende-se propor um modelo de otimização dos parâmetros do filtro sinc-janelado utilizando a função de janela Jacobi, além do intervalo de guarda, com o objetivo de minimizar a OOBE e maximizar a eficiência espectral e a SIR do sistema f-OFDM.

\section{REFERÊNCIAS}

[1] V. Stanković, "Adjustable high resolution window function," Electronics Letters, vol. 54, no. 13, pp. 827-829, 2018.

[2] J. Koester and J.-S. Chen, "Conforming window functions for meshfree methods," Computer Methods in Applied Mechanics and Engineering, vol. 347, no. 2019, pp. 588-621, 2019.

[3] J. Li, Z. Dong, L. Luo, S. Duan, and L. Wang, "A novel versatile window function for memristor model with application in spiking neural network," Neurocomputing, 2020.

[4] V. Vakilian, T. Wild, F. Schaich, S. ten Brink, and J. Frigon, "Universalfiltered multi-carrier technique for wireless systems beyond lte," in 2013 IEEE Globecom Workshops (GC Wkshps), 2013, pp. 223-228.

[5] J. Abdoli, M. Jia, and J. Ma, "Filtered ofdm: A new waveform for future wireless systems," in 2015 IEEE 16th International Workshop on Sig. Process. Advances in Wireless Comm. (SPAWC), 2015, pp. 66-70.

[6] X. Zhang, M. Jia, L. Chen, J. Ma, and J. Qiu, "Filtered-ofdm - enabler for flexible waveform in the 5th generation cellular networks," in 2015 IEEE Global Comm. Conf. (GLOBECOM), 2015, pp. 1-6.

[7] M. Yang, Y. Chen, and L. Du, "Interference analysis and filter parameters optimization for uplink asynchronous f-ofdm systems," IEEE Access, vol. 7, pp. 48 356-48 370, 2019.

[8] L. Jiang, H. Zhang, S. Cheng, H. Lv, and P. Li, "An overview of fir filter design in future multicarrier communication systems," Electronics, vol. 9, no. 4, 2020.

[9] X. Yang, S. Yan, X. Li, and F. Li, "A unified spectrum formulation for ofdm, fbmc, and f-ofdm," Electronics, vol. 9, no. 8, 2020.

[10] R. Ahmed, F. Schaich, and T. Wild, OFDM Enhancements for $5 G$ Based on Filtering and Windowing. Springer, 2019, pp. 39-61.

[11] F. Conceição, M. Gomes, V. Silva, R. Dinis, A. Silva, and D. Castanheira, "A survey of candidate waveforms for beyond $5 \mathrm{~g}$ systems," Electronics, vol. 10, no. 1, 2021

[12] S. Ahmadi, "Chapter 3 - new radio access physical layer aspects (part 1)," in 5G NR. Academic Press, 2019, pp. 285-409.

[13] F. J. Harris, "On the use of windows for harmonic analysis with the discrete fourier transform," Proceedings of the IEEE, vol. 66, no. 1, pp 51-83, 1978 .

[14] F. Di Stasio, M. Mondin, and F. Daneshgaran, "Multirate 5g downlink performance comparison for f-ofdm and w-ofdm schemes with different numerologies," in 2018 ISNCC, 2018, pp. 1-6.

[15] Huawei, "Link level evaluation results of waveform proposals," $3 G P P$ TSG RAN WG1 Meetin, R1-165426, 2016.

[16] J. Gautam, A. Kumar, and R. Saxena, "Windows: A tool in signal processing," IETE Technical Review, vol. 12, no. 3, pp. 217-226, 1995.

[17] J. Kaiser and R. Schafer, "On the use of the i0-sinh window for spectrum analysis," IEEE Transactions on Acoustics, Speech, and Signal Processing, vol. 28, no. 1, pp. 105-107, 1980.

[18] P. Lynch, "The dolph-chebyshev window: a simple optimal filter," Monthly Weather Review, vol. 125, pp. 655-660, 1997.

[19] M.-F. Tang and B. Su, "Joint window and filter optimization for new waveforms in multicarrier systems," EURASIP Journal on Advances in Signal Processing, vol. 63, no. 2018, pp. 1-20, 2018.

[20] M. R. P. da Silva and F. G. C. Rocha, "Proposta de janela baseada na distribuição de nakagami para o filtro fir utilizado na tecnologia f-ofdm candidata para aplicações 5g," in Simpósio Brasileiro de Telecomunicações e Processamento de Sinais, 2019, pp. 1-5.

[21] G. Szegö, Orthogonal Polynomials. American Math. Society, 1939.

[22] S. W. A. Bergen and A. Antoniou, "Generation of ultraspherical window functions," in 2002 11th European Sig. Process. Conf., 2002, pp. 1-4. 